\title{
Effect of lumbar support on human-head movement and discomfort in whole-body vibration
}

\author{
Jonathan DeShaw ${ }^{\mathrm{a}}$ and Salam Rahmatalla $\mathrm{a}^{\mathrm{a}, \mathrm{b}, *}$ \\ ${ }^{a}$ Center for Computer-Aided Design, The University of Iowa, Iowa City, IA, USA \\ ${ }^{\mathrm{b}}$ Department of Civil and Environmental Engineering, The University of Iowa, Iowa City, IA, USA
}

\begin{abstract}
.
BACKGROUND: It is well known that prolonged sitting can elicit low back pain as a result of the abnormal shape of the spine. This problem becomes worse in whole-body vibration environments where a rocking motion of the pelvis can occur and may amplify the vibration motion transmitted to the lumbar spine.

OBJECTIVE: Although the use of lumbar supports is common in static environments, little is known about how the use of such supports changes the biodynamic response and discomfort of the person in whole-body vibration environments.

METHODS: The motion at the head and the discomfort of ten participants were recorded under five back-support conditions, including three commercially available lumbar supports.

RESULTS: The results indicated significantly lower head motion and discomfort $(p<0.05)$ with the use of the lumbar supports than with a simple flat backrest.

CONCLUSIONS: All lumbar supports used in this study combined the advantages of reduced head motion and reduced discomfort during whole-body vibration.
\end{abstract}

Keywords: Lumbar support, whole-body vibration, heavy equipment seating

\section{Introduction}

It is well known that sitting for prolonged periods can elicit low back pain. During sitting, the lumbar disc will change shape when the spine curvature is flattened [1,2]. One way to maintain a more beneficial sitting posture is with the aid of a lumbar support. Generally, the lumbar support is placed between the L2-L5 vertebral bodies [3,4] and is made of foam or mesh. Lumbar supports have several advantages, such as supporting the pelvis, providing balance for the spinal column, and allowing relaxation of back muscles and intervertebral discs. Makhsous et al. [3] found that lumbar support resulted in reduced sitting load on the lumbar spine and reduced the lumbar muscular activity, which may potentially reduce sitting-related low back pain. In fact, disc pressure was found to be lower with a lumbar support than without a support regardless of backrest angle [5,6]. One particular study investigated how the iliolumbar ligaments are loaded during slouching as it relates to low back pain [7]. The results of the study showed

\footnotetext{
${ }^{*}$ Corresponding author: Salam Rahmatalla, Department of Civil and Environmental Engineering, The University of Iowa, 4121 Seamans Center, Iowa City, IA 52242, USA. E-mail: salam-rahmatalla@uiowa.edu.
} 
that the recommended posture was a backrest with lumbar support with free shoulder space, where the shoulder blades are not in direct contact with a backrest surface.

In a whole-body vibration (WBV) environment, such as that experienced while operating heavy mobile equipment, an additional rocking motion of the pelvis can occur and may amplify the vibration motion transmitted to the spine [8]. Wilder et al. [8] indicated that an increase in lumbar support causes lordosis, reducing the deformation of the lumbar spine and therefore reducing the associated disc pressure. Examination of operators with at least ten years of exposure to WBV showed that morphological changes in the lumbar spine occurred earlier and much more frequently than in the general population [9]. Although researchers have concluded that lumbar support should be one of the main factors considered in cab and seat designs [10,11], to the authors' knowledge, no one has taken into account how lumbar support may change the biodynamic response of the operator in WBV environments.

The effect of vibration exposure on human health and comfort in many occupations where operators are seated for prolonged periods is well known. Positive correlations between biomechanical responses and subjective perception and the amount of vibration transferred to the participants have been found in recent studies [12-15]. The current standard for WBV (ISO 2631-1) [16] can give guidance about human health and discomfort, but it is measured at the seat/human interface and therefore is incapable of quantifying differences between human postures in WBV [17]. The total energy transferred through the bodies of operators due to vibration is of great interest, especially when changing postures or seatsupporting conditions [18]. Researchers generally agree on the potential of seat-to-head transmissibility (STHT) in capturing the perception of vibration of seated people [19-21]. This metric quantifies the amount of vibration energy transferred through the body $[19,20,22,23]$ and can be used as a measure of effectiveness for seat design [24-26].

It appears that studies on WBV agreed that motion at the head and inappropriate back support are two major contributors to the operator's short- and long-term health and performance. While the importance of lumbar supports for comfort in static sitting postures is known, little is known about the dynamic effects of these supports in WBV environments. This work hypothesizes that lumbar supports will reduce the head motion due to input WBV while providing comfort and potentially better protection to the operator. The hypothesis was tested on ten participants by comparing their head accelerations and discomfort levels under multi-axis WBV vibration using five different back-support conditions, including three commercially available lumbar supports.

\section{Methods}

\subsection{Participants}

Ten male participants with an average age of $23.7 \pm 2.3$ years, height of $180.3 \pm 4.4 \mathrm{~cm}$, and weight of $88.5 \pm 11.1 \mathrm{~kg}$ took part in this study. All participants signed a consent form approved by the University of Iowa Institutional Review Board. The University of Iowa Institutional Review Board reviewed and approved the ethics of this research.

\subsection{Experimental seating setup}

Five back-support conditions were investigated in this work: sitting upright (no backrest) (Fig. 1(a)), using a rigid flat backrest (Fig. 1(b)), and using three types of commercially available lumbar supports (Figs 1(c) and 2). All seating conditions, including lumbar support conditions, were randomized for 


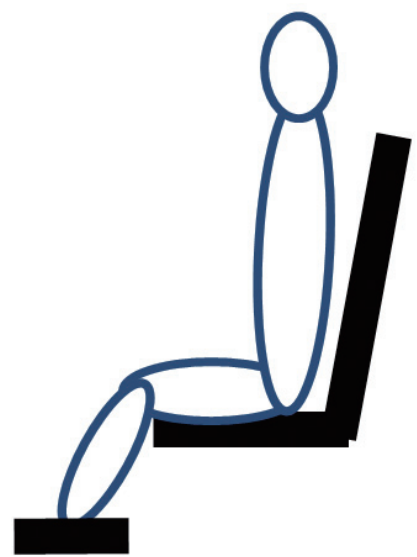

(a) No Backrest

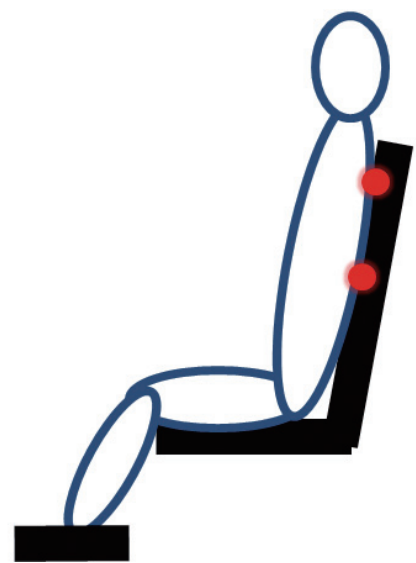

(b) Flat Backrest

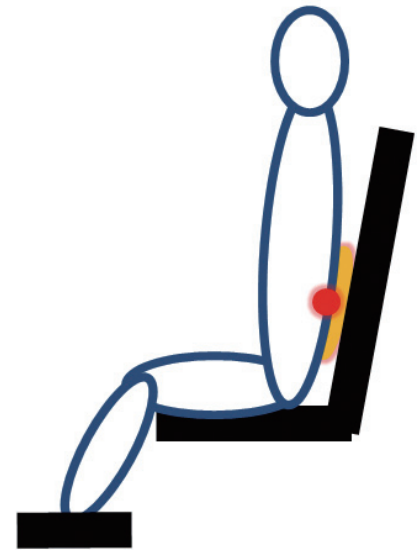

(c) Lumbar Backrest

Fig. 1. Three seating configurations used in study: (a) no backrest support, (b) flat backrest support, (c) lumbar backrest support. Dots indicate backrest contact points, and light-colored ovals represent the lumbar support location.

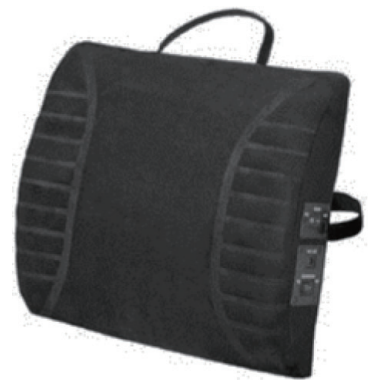

(a) LS1

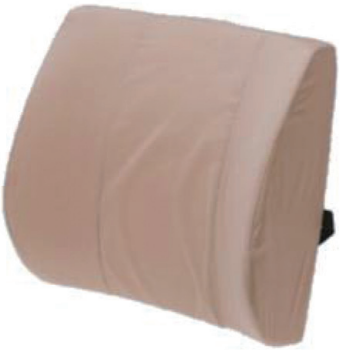

(b) LS2

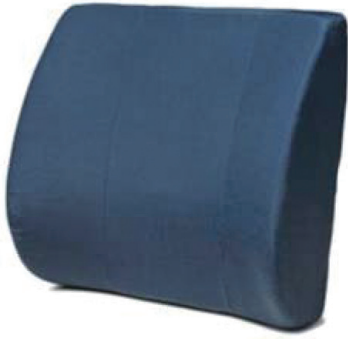

(c) LS3

Fig. 2. The lumbar backrest support condition used three commercially available lumbar support types: (a) black cushion, convex type, soft foam support LS1, (b) tan cushion, concave type, hard foam, support LS2, and (c) blue cushion, concave type, soft foam, support LS3.

each participant. The participants were seated in a rigid seat that was coated with thin rubber and rigidly mounted to a vibration platform. The thin rubber was used to increase friction and had negligible WBV effects on the participants. A footrest on the platform was adjusted so that the participants were comfortable and their thighs were approximately horizontal.

The tan lumbar support (LS2) and blue lumbar support (LS3) (Figs 2(b) and 2(c)) had a similar shape, with sides that seemed to "hug" the individual's back, while the black support (LS1) (Fig. 2(a)) had a simpler "open" shape. LS2 was made of a higher-density foam, and LS1 and LS3 were made of a softerdensity foam. Each lumbar support had a flat posterior surface that rested against the flat seatback during testing, and each participant was instructed to adjust the vertical position of the lumbar support so that it was placed at the deepest part of their lumbar curve (approximately at L2-L5) prior to each trial.

\subsection{Vibration simulation}

Participants were exposed to multiple-axis (3D) random vibration stimuli from 0.5 to $12 \mathrm{~Hz}$ at a 1.8 $\mathrm{m} / \mathrm{s}^{2} \mathrm{RMS}$ resultant acceleration magnitude ( $1.08 \mathrm{~m} / \mathrm{s}^{2} \mathrm{RMS}$ in each direction). The vibration files were 

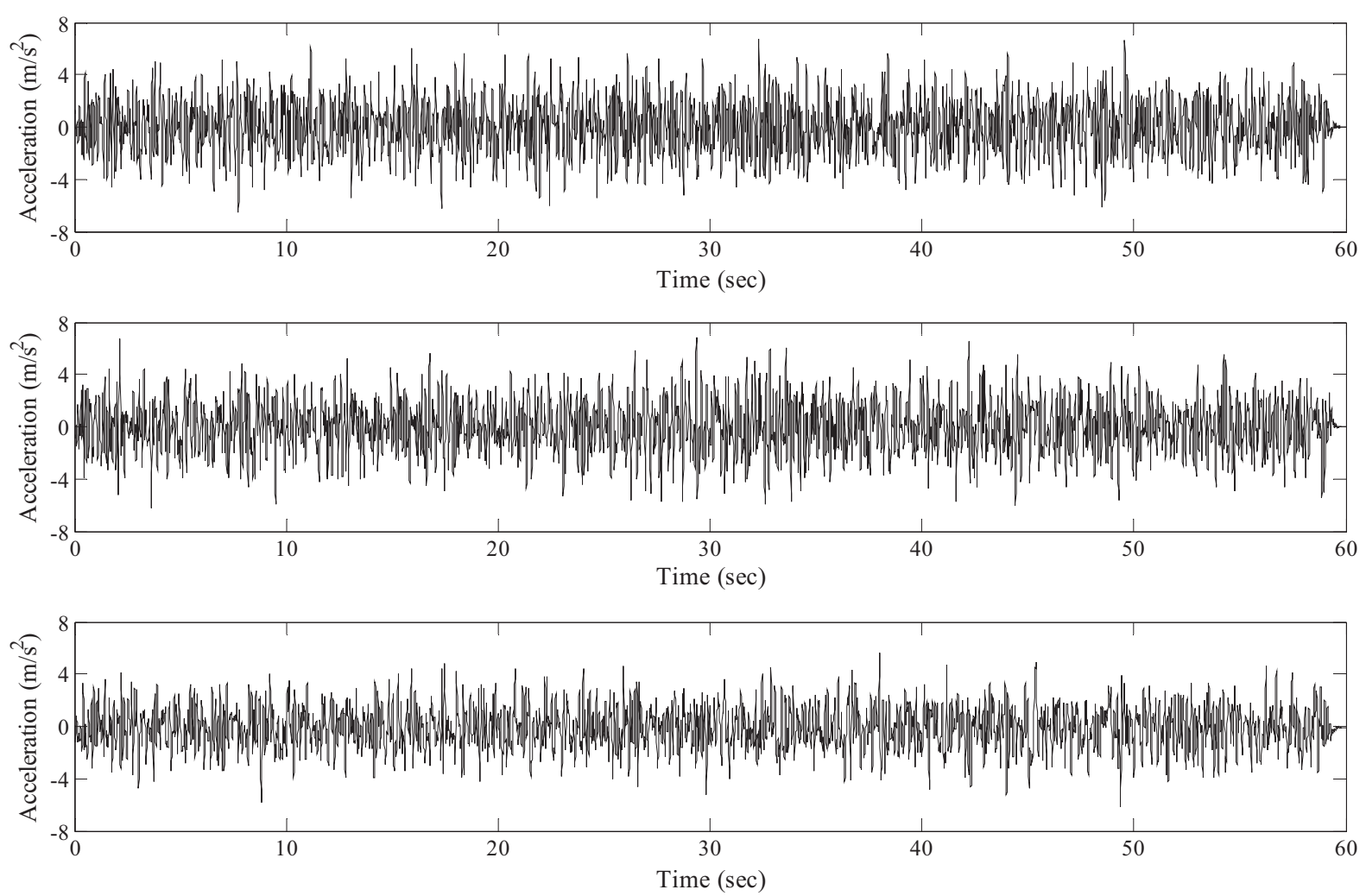

Fig. 3. Acceleration profiles of the multiple-axis (3D) random vibration used in the study. From top to bottom: fore-aft vibration component, lateral vibration component, and vertical vibration component.

created from white noise and band-pass filtered to achieve the desired frequency range. Each signal was randomized in each of the three directions simultaneously. While many ground vehicle profiles can be replicated in a controlled laboratory setting [27], broadband vibrations provide a more general test that covers a larger range of human body response and is applicable to many different vehicles. Also, using the broadband signal in a 3D multi-axis environment allows the characterization of a human system in three directions at once [28]. Figure 3 shows the axial components of the acceleration profiles used in the study. A six-degree-of-freedom human-rated motion simulator (Moog-FCS 628 electrical system, Ann Arbor, MI) was used to produce ride files with 60 seconds of vibration exposure (Fig. 4). The motion simulator was able to reproduce accurately the given signal for each participant with RMS error of less than $1 \%$ of the signal.

\subsection{Discomfort data collection}

For each type of seating condition, the participants were asked to rate their discomfort on the Borg CR10 scale [29] during vibration. The Borg CR-10 scale is an absolute scale that ranges from 0 to 10 , with 0 indicating no discomfort and 10 indicating extremely strong discomfort. The scale has accompanying keywords to assist the user. Because the scale is an absolute scale, it allows for comparison between multiple postures and vibration settings. The participant-reported discomfort data were then normalized by the participant's maximum rating; this was done because each participant used the scale differently, and the goal was to find trends between the conditions only. 


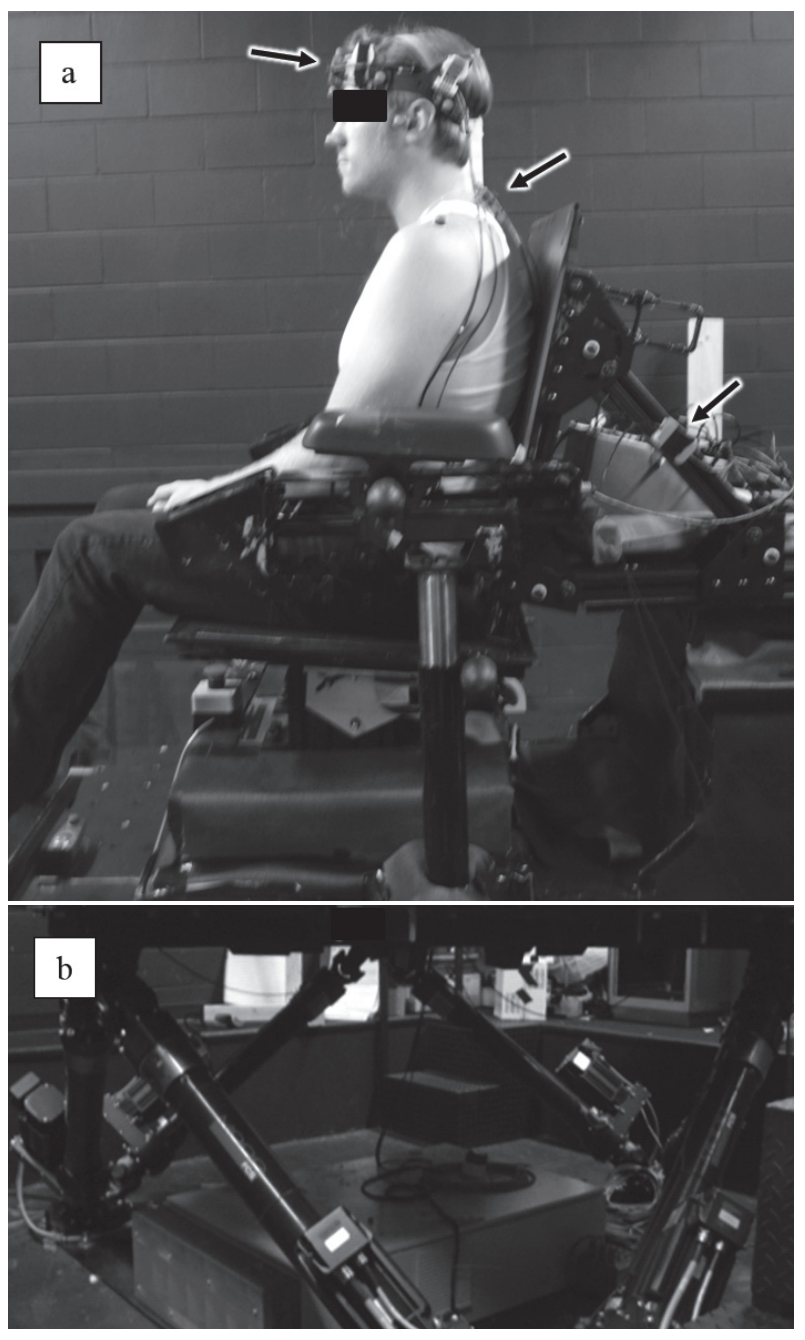

Fig. 4. Experimental setup: (a) participant assuming no backrest posture, and (b) motion simulator base used to generate multi-axis vibrations. Arrows in (a) show the locations of the MTx sensors.

Each participant was instructed to verbally rate his discomfort towards the end of the 60-second WBV ride file. A previous study [30] found that to be sufficient time for the participants to rate the exposure. Each participant was asked to rate his discomfort from his upper body only, not considering the legs or the comfort of the seat pan itself. The sequences were randomized for each participant.

\subsection{Sensor data collection}

Accelerometers are traditionally considered as the main measurement tools in WBV studies, but their measurements become questionable when they are attached to inclined surfaces, such as the contours of the human body, or when the motion has multiple directions. To circumvent this problem, inertial sensors were used in this work, and the acceleration data were corrected as described by DeShaw and Rahmatalla [31]. 
The participants wore an inertial sensor at the forehead region (MTx inertial tracker, Xsens Technologies, Enschede, Netherlands) (Fig. 4), which was rigidly attached to a head-worn halo similar to that used in previous studies [31,32]. An additional sensor was rigidly attached to the aluminum seat frame to measure the input motion to the human. Each inertial sensor had the capability to record the acceleration data at $120 \mathrm{~Hz}$ and the orientation matrix of the sensor local coordinate system relative to the global coordinate system. The data obtained from the inertial sensors were verified with a traditional tri-axial DC accelerometer (Dytran 7523A1, Chatsworth, CA) as outlined by DeShaw and Rahmatalla [31]. Additional retroreflective markers were attached to the participants to capture motion data; however, the data were not used in this work.

\subsection{Seat-to-head transmissibility}

The energy transfer through the body represented by the seat-to-head transmissibility (STHT) was used in this work to measure head motion that occurred as a result of the input vibration at the seat level. The STHT is normally defined as shown in Eq. (1).

$$
\operatorname{STHT}_{X x}(\omega)=S_{X X}^{-1}(\omega) S_{X x}(\omega)
$$

where $S_{X x}$ represents the cross-spectral density between the input motion (X-direction) at the seat level and the output motion (x-direction) at the head level, and $S_{X X}$ represents the auto-spectral density of the input motion $(X)$. For multi-axis input/output, Eq. (1) takes a matrix form as shown in Eq. (2) [33], where the input has three components in the three Cartesian directions represented by uppercase letters: fore-aft $(X)$, lateral $(Y)$, and vertical $(Z)$, and the output has three directions of motion represented by lowercase letters: fore-aft $(x)$, lateral $(y)$, and vertical $(z)$.

$$
\left[\begin{array}{lll}
S T H T_{X x} & S T H T_{X y} & S T H T_{X z} \\
S T H T_{Y x} & S T H T_{Y y} & S T H T_{Y z} \\
S T H T_{Z x} & S T H T_{Z y} & S T H T_{Z z}
\end{array}\right]=\left[\begin{array}{lll}
S_{X X} & S_{X Y} & S_{X Z} \\
S_{Y X} & S_{Y Y} & S_{Y Z} \\
S_{Z X} & S_{Z Y} & S_{Z Z}
\end{array}\right]^{-1}\left[\begin{array}{ccc}
S_{X x} & S_{X y} & S_{X z} \\
S_{Y x} & S_{Y y} & S_{Y z} \\
S_{Z x} & S_{Z y} & S_{Z z}
\end{array}\right]
$$

\subsection{Statistical analysis of variance (ANOVA)}

Statistical analyses were performed by using one-way ANOVA in conjunction with a multiple comparisons tests (Bonferroni method) between the means of the 10 participants during the five seating conditions. These analyses were performed at each time step of the seat-to-head-transmissibility so that the ranges along the frequency axis could be determined to be statistically significant with a cut-off $p$ value of 0.05 .

\section{Results}

\subsection{Effect of lumbar supports on seat-to-head transmissibility}

As shown in Fig. 5, the transmissibility matrix in multi-axis input/output vibration has nine components. In most WBV studies, the common input/output combination of interest is the vertical input to vertical output response $(\mathrm{Zz})$. Obvious changes are seen in the vertical direction depending on the backrest and lumbar support conditions. The lowest STHT occurred with the no-backrest posture. Each of the three lumbar supports changed the characteristics of the system and showed an STHT peak at around 4-5 Hz, with a secondary peak around $10 \mathrm{~Hz}$. The magnitude of the STHT using any of the lumbar 

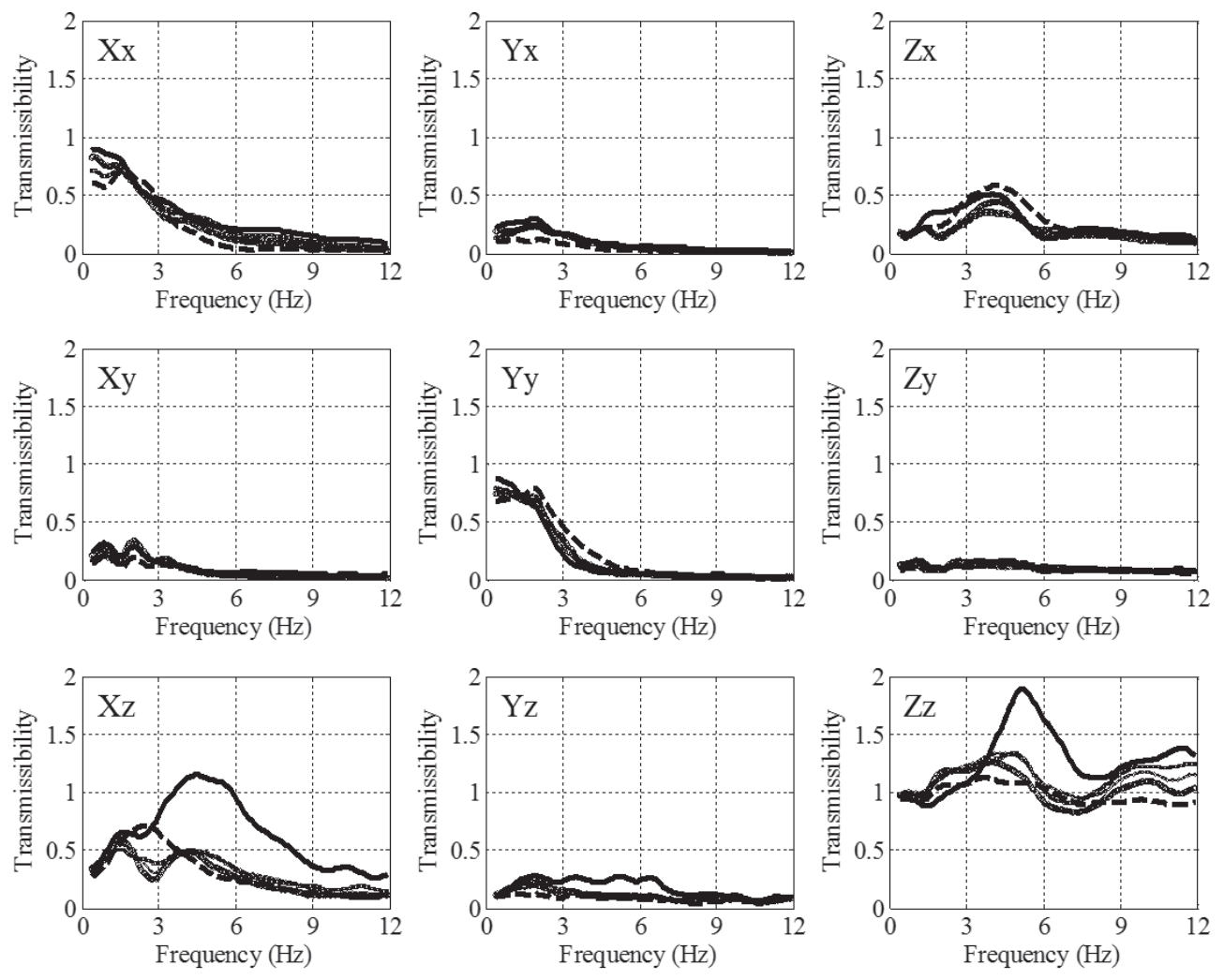

--- No Backrest

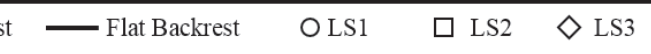

Fig. 5. Average seat-to-head transmissibility components (three input directions with three output directions) for five seating and lumbar support conditions.

supports was greatly reduced from that of a flat backrest, with an average reduction from 1.9 to 1.4. This reduction was statistically significant below $3.5 \mathrm{~Hz}$ and above $4.5 \mathrm{~Hz}$ with $p<0.05$.

Other transmissibility components of interest are the vertical input to fore-aft output (Zx) and fore-aft input to vertical output $(\mathrm{Xz})$, as these terms generally relate to the pitch of the head. The Xz component was relatively larger for the flat backrest condition at frequencies above $2.5 \mathrm{~Hz}(p<0.05)$, while minimal differences were seen in the Zx component between the different lumbar support conditions. The fore-aft to fore-aft (Xx) component showed a slight change between conditions, where the highest magnitude occurred for the flat backrest condition followed by the lumbar support conditions; however, the differences were statistically insignificant $(p>0.05)$. The no-backrest condition had a reduced transmissibility compared to the backrest condition at frequencies greater than $5 \mathrm{~Hz}(p<0.05)$, but its peak shifted from low frequency $(<0.5 \mathrm{~Hz})$ to around $1.5 \mathrm{~Hz}$. The lateral-to-lateral (Yy) component showed little change between the conditions, except between the no-backrest condition compared to the rest of the conditions in the range of $2-6 \mathrm{~Hz}(p<0.05)$.

Figure 6 displays the variability of the participants' transmissibility in the vertical direction (Zz) under the different conditions. A relatively high variability is seen during the no-backrest posture compared to the other postures. A few of the participants showed relatively large variability around the first peak, especially with supports LS2 and LS3. 


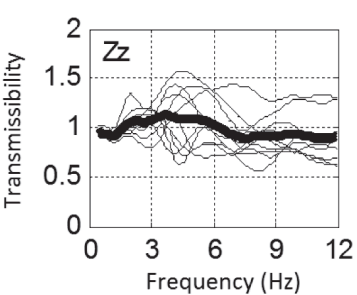

(a) No Backrest

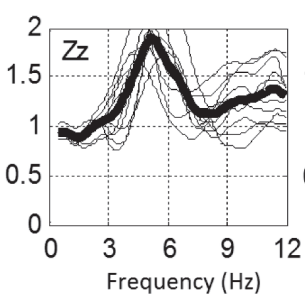

(b) Flat Backrest

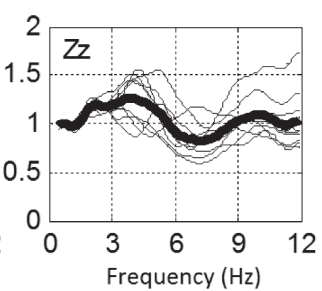

(c) LS1

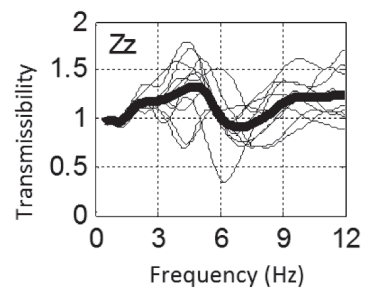

(d) LS2

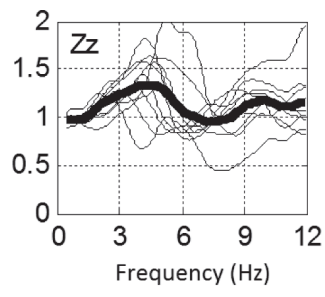

(e) LS3

Fig. 6. Vertical seat-to-head transmissibility for five seating and lumbar support conditions. The thin lines represent the individual participants, while the bold line represents the average.

\subsection{Effective seat-to-head transmissibility}

As shown in Eq. (2) and Fig. 5, the transmissibility matrix in multi-axis input/output vibration has nine components, making it much harder to deal with and infer information from than the single-axis input/output vibration. In a previous work [34], the authors developed a new measure called the effective seat-to head transmissibility (ESTHT), where they transform the nine components of the multi-axis STHT into one component, similar to that of a single-axis input/output vibration. This mathematical operation is done by taking the single value decomposition of the nine components and then using the maximum distortion energy theory [35] to calculate a resultant in the frequency domain.

When using the ESTHT, the nine components of the STHT were transformed to one component similar to that of the single-axis input/output vibration in an objective manner. Figure 7 shows that the greatest energy transmitted from the seat to the head occurred with the flat-backrest posture, while the lowest total energy to the head occurred with the no-backrest posture. This difference in magnitude was statistically significant below $2 \mathrm{~Hz}$ and above $3 \mathrm{~Hz}(p<0.05)$. The use of the backrest with lumbar supports shifted the resonance frequency (the peak) slightly less than the flat backrest; however, the amplitude was decreased at the peak resonance area of 4.5-7 Hz with $p<0.05$. Also, the first lumbar support (LS1) had the lowest-resonance peak; however, no significant differences were found between lumbar support types. The plots in Fig. 7 show that the foam difference between supports LS2 and LS3 had little effect on the ESTHT.

\subsection{Effect of lumbar support on reported discomfort}

The participants rated their discomfort based on upper-body comfort on the Borg CR-10 scale (Fig. 8). This discomfort rating took into account the vibration transmission to the head as well as comfort from the backrest condition. The ratings were then normalized by dividing by each participant's maximum value to give insights into the participants' evaluation of the different seated conditions. There was a very high variability among the participants, but some general trends were seen. The participants rated the first lumbar-support condition the most comfortable of all the postures, while the flat backrest was reported as 


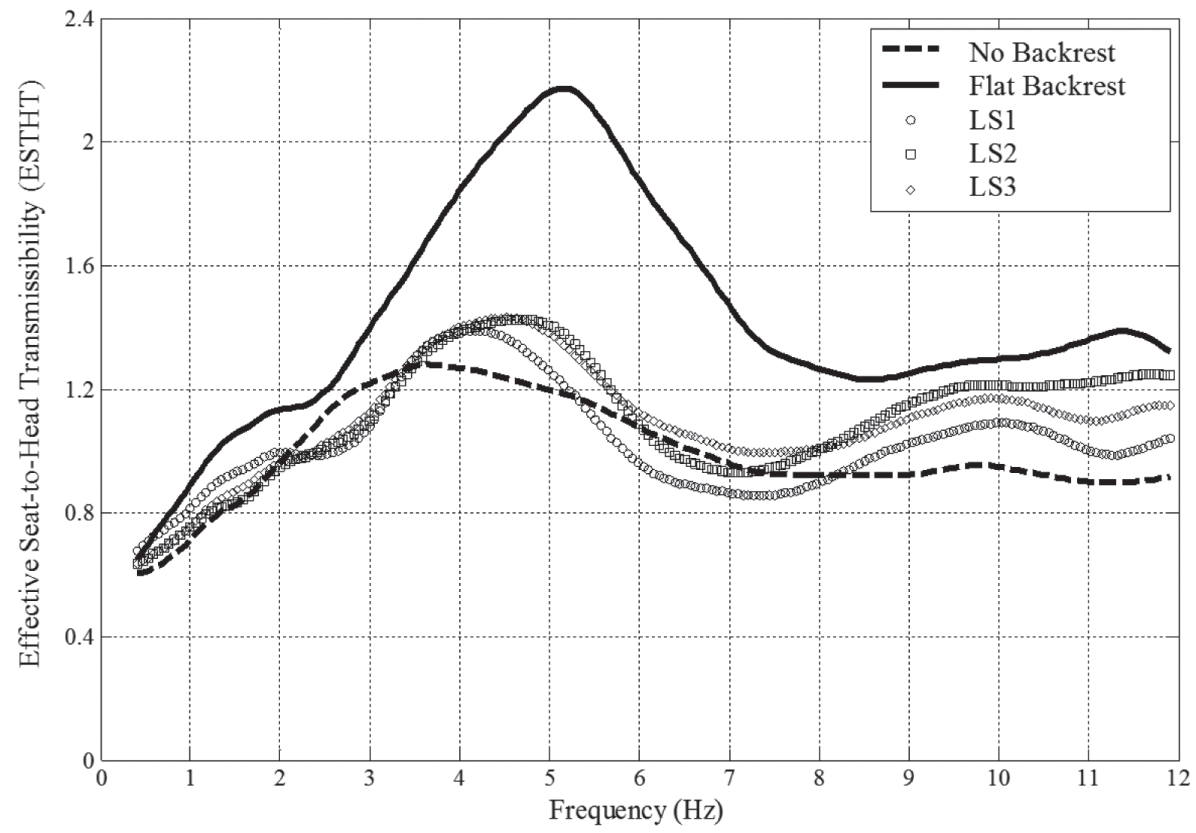

Fig. 7. The mean effective seat-to-head transmissibility of ten participants under multi-axis input/output vibration using five seating conditions.

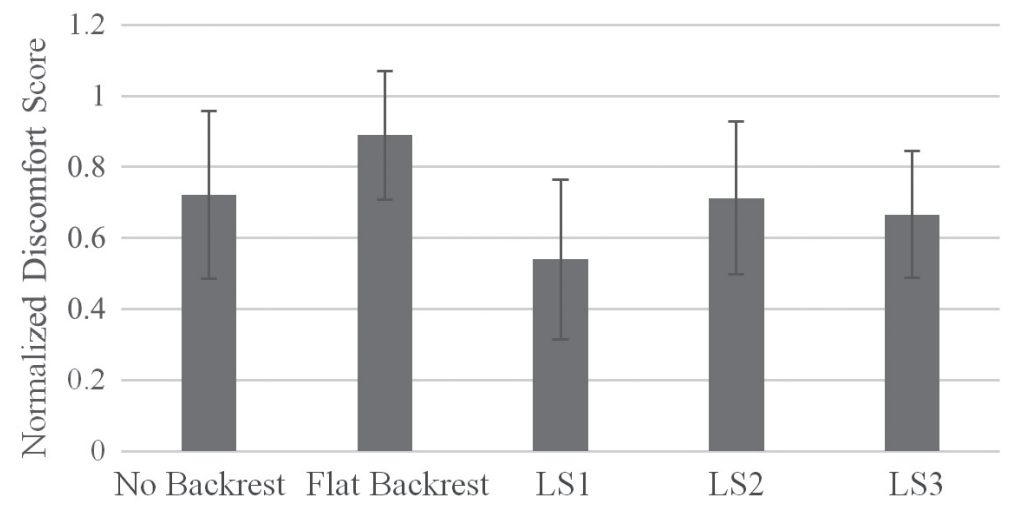

Fig. 8. The normalized discomfort score in the five seating conditions. Average and standard deviation of 10 participants.

the most uncomfortable $(p<0.05)$. The lumbar-support conditions were all rated as more comfortable than the flat backrest, but there was little difference between supports LS2 and LS3. The discomfort experienced by the participants is likely linked to the comfort of the seat and the uncomfortable motions experienced during the simulation. The no-backrest condition was actually more comfortable than the flat backrest but less comfortable than support LS1.

\section{Discussion}

This work demonstrated that the greatest factor in the vibration transmission to the head and discomfort during WBV is simply whether or not a backrest is used at all. The results showed that the vibration 
transmission to the head was substantially lower $(p<0.05)$ below $2 \mathrm{~Hz}$ and above $3 \mathrm{~Hz}$ in the nobackrest condition than in the flat back-support condition. Although this may seem positive, during the no-backrest seating condition, operators are required to use their core muscles to stabilize themselves, leading to greater fatigue, which may lead to awkward postures that may generate undesired health consequences $[1,36]$. Additionally, with the no-backrest condition, there is no guarantee that the lordotic curve is being maintained. Thus, having the back comfortably supported in the correct manner with reduced head motion can be considered the best configuration for a seated position under WBV.

In terms of the motion at the head, the results showed that the peak magnitude of the STHT is significantly reduced $(p<0.05)$, especially in the vertical direction, when using any of the lumbar-support conditions compared to the flat-backrest condition. This is likely due to the lack of contact between the shoulder region and the seatback in the flat-backrest condition. This finding is consistent with the finding of Snijders et al. [7], who recommended a lumbar support with free shoulder space.

While the STHT can partially capture the energy transfer to the participant's head, it may not capture the energy transferred from other directions. In this case, the results showed that the ESTHT will objectively sum all of the energy coming to the head from the different directions at each frequency and therefore may provide a more realistic picture of the actual energy transferred to the head. For example, the vertical component of the STHT of the flat backrest in Fig. 5, which is commonly used by researchers, has a peak magnitude of 1.9 and occurred at $5.2 \mathrm{~Hz}$; the ESTHT of Fig. 7, on the other hand, has a peak magnitude of 2.2 at $5.2 \mathrm{~Hz}$. This indicates that in multi-axis WBV, the STHT in the vertical direction will underestimate the resulting pitch motion at the head. The reduced head pitch motion due to the support conditions may be highly beneficial to machinery operators' vision and performance, especially as more electronics are used in such vehicles.

The results also showed an obvious positive contribution of lumbar support on the participants' reported discomfort. The addition of the lumbar support cushion gave the participants greater comfort, as can be seen by the subjective rating of the participants under the different testing conditions in Fig. 8 . This is expected to be more important as the duration of vibration increases. Overall, the lumbar supports seemed to offer backrest support and comfort while reducing head motion. The difference between a flat backrest and lumbar support was pronounced, where the flat backrest provides more support to the upper-back and the shoulder areas, and allows more energy to transfer to the head [37]; therefore, introducing more head motion. The lumbar supports, on the other hand, add support to the lumbar and the pelvis areas that may result in reduced pelvis motion and a more stable lumbar spine with a natural shape.

The types of lumbar support used in this work seemed to have very little effect. The two supports that were of the same shape and dimensions (LS2 and LS3) had little noticeable difference on the transmission of vibration to the head. The black lumbar support (LS1) had a slight (but not statistically significant with $p>0.05$ ) change in magnitude and peak frequency, most likely due to the shape of the support. The material types and shapes of the lumbar support may have influence on the resulting head motion and discomfort levels; however, in this work limited types of supports were used. Therefore, a more detailed study may provide specific guidelines as to the most effective materials and shapes for the lumbar support.

\section{Conclusions}

Overall, the lumbar supports combined the advantages of lower head motion and lower discomfort than that found with a flat backrest. Also, it is expected that lumbar support would help keep the natural shape of the lumbar spine during operations and would help operators avoid awkward postures that 
may lead to unpleasant consequences [1]. Furthermore, the literature is consistent in noting that lumbar support is effective in keeping the lordotic shape in the static seated posture. Therefore, operators can sit with a back support at the most critical area of the back, the lumbar spine, while reducing head motion. Although the importance of lumbar supports is widely recognized, seat designers need to better understand how lumbar supports change the human biodynamic response in vibration environments. Better seat and support designs will lead to a reduction in musculoskeletal disorders among heavy machinery operators.

\section{Conflict of interest}

The authors have no conflict of interest to report.

\section{References}

[1] D.G. Wilder and M.H. Pope, Epidemiological and aetiological aspects of low back pain in vibration environments - an update, Clinical Biomechanics, 11(2) (1996), pp. 61-73.

[2] G. Anderson, R.W. Murphy, R. Orengren and A.L. Nachemson, The influence of Backrest Inclination and Lumbar Support on Lumbar Lordosis, Spine, 4(1) (1979).

[3] M. Makhsous, F. Lin, J. Bankard, R.W. Hendrix, M. Hepler and J. Press, Biomechanical effects of sitting with adjustable ischial and lumbar support on occupational low back pain: evaluation of sitting load and back muscle activity, $B M C$ Musculoskeletal Disorders, 10(17) (2009).

[4] N. Coleman, B.P. Hull and G. Ellitt, An empirical study of preffed settings for lumbar support on adjustable chairs, Ergonomics, 41(4) (1998), pp. 401-419.

[5] V.D. Bhise, Ergonomics in the Automotive Design Process, Boca Raton, FL: CRC Press, 2011

[6] D.B. Chaffin, G. Andersson and B.J. Martin, Occupational Biomechanics, New York: John Wiley and Sons, 1999.

[7] C.J. Snijders, P.F. Hermans, R. Niesing, C.W. Spoor and R. Stoeckart, The influence of slouching and lumbar support on iliolumbar ligaments, intervertebral discs and sacroiliac joints, Clinical Biomechanics, 19(2004), pp. 323-329.

[8] D.G. Wilder, M.H. Pope and M. Magnusson, Mechanical Stress Reduction During Seated Jolt/Vibration Exposure, Seminars in Perinatology, 20(1) (1996), pp. 54-60.

[9] H. Dupuis and G. Zerlett, Whole-body vibration and disorders of the spine, Int Arch Occup Environ Health, 59(1987), pp. 323-336.

[10] N.K. Kittusamy, A Checklist for Evaluating Cab Design of Construction Equipment, Applied Occupational and Environmental Hygiene, 18(10) (2003), pp. 721-723.

[11] Z. Srdjevic, Operators' Participation in Selecting the Most Ergonomic Driver Seat, Journal of Agricultural Safety and Health, 11(3) (2005), pp. 347-352.

[12] M.J. Griffin, Discomfort from feeling vehicle vibration, Vehicle System Dynamics, 45(2007), pp. 679-698.

[13] S. Maeda, N.J. Mansfield and N. Shibata, Evaluation of subjective response to whole-body vibration exposure: Effect of frequency content, International Journal of Industrial Ergonomics, 38(2008), pp. 509-515.

[14] M. Schust, R. Bluthner and H. Seidel, Examination of perceptions (intensity, seat comfort, effort) and reaction time (brake and accelerator) during low-frequency vibration in $\mathrm{x}$ - or $\mathrm{y}$-direction and biaxial (xy-) vibration of driver seats with activated and deactivated suspension, Journal of Sound and Vibration, 298(2006), pp. 606-626.

[15] G. Subashi, N. Nawayeh, Y. Matsumoto and M.J. Griffin, Nonlinear subjective and dynamic responses of seated subjects exposed to horizontal whole-body vibration, Journal of Sound and Vibration, 321(2009), pp. 416-434.

[16] I.S. Organization, Mechanical vibration and shock - Evaluation of human exposure to whole-body vibration - Part 1: General Requirements, 1997

[17] J. DeShaw and S. Rahmatalla, Predictive discomfort in single- and combined-axis whole-body vibration considering different seated postures, Human Factors, 56(5) (2014), pp. 850-863.

[18] J. DeShaw and S. Rahmatalla, Effective Seat-to-head Transmissibility under Combined-Axis Vibration and Multiple Postures, Int. J. Vehicle Performance, 1(3/4) (2014).

[19] M. Griffin, Handbook of Human Vibration, London: Academic Press, 1990.

[20] M. Demic and J. Lukic, Investigation of the transmission of fore and aft vibration through the body, Applied Ergonomics, 40(2009), pp. 622-629. 
[21] G.S. Paddan and M.J. Griffin, A review of the transmission of translational seat vibration to the head, Journal of Sound and Vibration, 21(1998), pp. 863-882.

[22] W. Wang, S. Rakheja and P.E. Boileau, Relationship between measured apparent mass and seat-to-head transmissibility responses of seated occupants exposed to vertical vibration, Journal of Sound and Vibration, 314(2008), pp. 907-922.

[23] R.J. Jack and T. Eger, The effects of posture on seat-to-head Whole-body vibration transmission, Journal of Low Frequency Moise, Vibration and Active Control, 27(4) (2008), pp. 309-325.

[24] J.L. Niekerk, W.J. Pielemeier and J.A. Greenberg, The use of seat effective amplitude transmissibility (SEAT) values to predict dynamic seat comfort, Journal of Sound and Vibration, 260(2003), pp. 867-888.

[25] A. Westhuizen and J.L. Niekerk, Verification of seat effective amplitude transmissibility (SEAT) value as a reliable metric to predict seat comfort, Journal of Sound and Vibration, 295(2006), pp. 1060-1075.

[26] Y. Qiu and M.J. Griffin, Transmission of fore-aft vibration to a car seat using field tests and laboratory simulation, Journal of Sound and Vibration, 264(2003), pp. 135-155.

[27] J.P. Dickey, T.R. Eger and M.L. Oliver, A systematic approach to simulating field-based occupational whole-body vibration exposure in the lab using a $6 \mathrm{df}$ robot, Work, 35(1) (2010), pp. 15-26.

[28] B. Hinz, G. Menzel, R. Bluthner and H. Seidel, Seat-to-head Transfer Function of Seated Men - Determination with Single and Three Axis Excitations at Different Magnitudes, Industrial Health, 48(2010), pp. 615-627.

[29] G. Borg, Psychophysical bases of perceived exertion, Medicine and Science in Sports and Exercise, 14(5) (1982), pp. 377-381.

[30] J.P. Dickey, M.L. Oliver, P.E. Boileau, T.R. Eger, L.M. Trick and A.M. Edwards, Multi-axis sinusoidal whole-body vibrations: Part I - How long should the vibration and rest exposures be for reliable discomfort measures? Journal of Low Frequency Noise, Vibration and Active Control, 25(2006), pp. 175-184.

[31] J. DeShaw and S. Rahmatalla, Comprehensive measurement in whole-body vibration, Journal of Low Frequency Noise, Vibration and Active Control, 31(2) (2012), pp. 63-74.

[32] W. Wang, S. Rakheja and P. Boileau, Effect of back support condition on seat to head transmissibilities of seated occupants under vertical vibration, Journal of Low Frequency Noise, Vibration and Active Control, 25(2006), pp. 239-259.

[33] D.E. Newland, An Introduction to random vibrations and spectral analysis, New York: Longman Inc., 1984.

[34] S. Rahmatalla and J. DeShaw, Effective seat-to-head transmissibility in whole-body vibration: Effects of posture and arm position, Journal of Sound and Vibration, 330(25) (2011), pp. 6277-6286.

[35] R.C. Hibbeler, Mechanics of Materials, Seventh Edition, New Jersey: Pearson Prentice Hall, 2008.

[36] N.J. Mansfield, J. Mackrill, A.N. Rimell and S.J. MacMull, Combined Effects of Long-Term Sitting and Whole-Body Vibration on Discomfort Onset for Vehicle Occupants, Automotive Engineering, 2014(2014), pp. 1-8.

[37] S. Rahmatalla, R. Smith, J. Meusch, T. Xia, T. Marler and M. Contratto, A Quasi-static discomfort measure in wholebody vibration, Industrial Health, 48(5) (2010), pp. 645-653. 